\title{
MEANS AND VARIANCES IN STOCHASTIC MULTISTAGE CANCER MODELS
}

\author{
AIDAN SUDBURY, ${ }^{*}$ Monash University
}

\begin{abstract}
A widely used model of carcinogenesis assumes that cells must go through a process of acquiring several mutations before they become cancerous. This implies that any time there will be several populations of cells at different stages of mutation. In this paper we give exact expressions for the expectations and variances of the number of cells in each stage of such a stochastic multistage cancer model .
\end{abstract}

Keywords: Multistage cancer model; mutation chain

2010 Mathematics Subject Classification: Primary 60J20; 92B05

\section{Introduction}

One model of cancer development that has been widely studied is what is generally called a stochastic multistage model (see, for example, Zheng (2008)). At a fixed rate a healthy cell, when it divides, may give rise to a healthy cell and a mutant cell. A mutant cell, when it divides, may then give birth to two mutant cells of the same type, or possibly to one of the same type and to one with a further mutation. This process may continue so that the population consists of several subpopulations of cells with each subpopulation having a different number of mutations. We represent such a model in Figure 1, where cell death has also been allowed for. The classical treatment of the two-stage model is given in Armitage and Doll (1957). However, it is now generally considered that the number of stages through which the cells have to pass before becoming carcinogenic is greater than two. Such models have been analysed using computer simulation in Connolly and Kimbell (1994) and theoretically in Portier et al. (1996), (2000). Previous derivations have been somewhat difficult to follow due to the mathematical complexity of the treatment. In this paper we consider a slightly simplified version of this model, allowing us to calculate exactly expectations and variances for the number of cells in each stage of the multistage model. Since it is believed that a certain number of mutations may be necessary before a tumour becomes cancerous, we shall also give a recursive formula for the probability that at time $t$ the number of mutations has reached a certain threshold.

The major drawback of this treatment is the assumption that all cells act independently of all others, an assumption that may be reasonable while the numbers of cancerous cells are small. Nor does the treatment take into account programmed cell death or the possibility that cancer rates may change at different ages.

We imagine that there are $r+1$ subpopulations, $S_{0}, S_{1}, \ldots, S_{r}$, and that the number of mutations in $S_{i}$ is $i$. Subpopulation $S_{0}$ is different from the rest. Otherwise, cells in subpopulation $S_{i}, i \neq 0$, divide in two ways. First, a cell is replaced by two new cells at rate $\mu_{i}$ so that the subpop- ulation increases by 1 . This can be considered a pure birth process at rate $\mu_{i}$.

Received 11 March 2011; revision received 28 October 2011.

* Postal address: School of Mathematical Sciences, Monash University, PO Box 28M, VIC 3800, Australia.

Email address: aidan.sudbury@monash.edu 


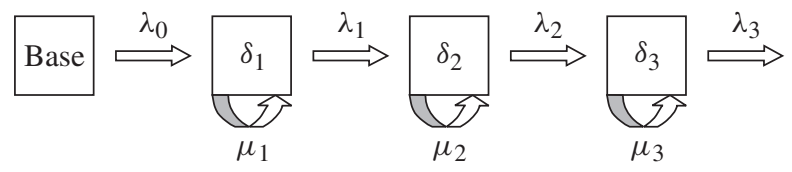

Figure 1.

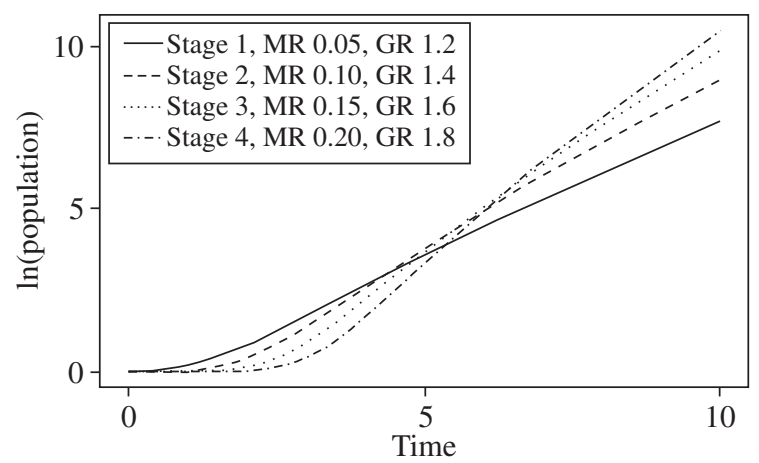

FIGURE 2: Log population size versus time for a four-stage model with mutation rates (MRs) $0.05-0.20$ and growth rates (GRs) 1.2-1.8.

Second, at rate $\lambda_{i}$, two new cells are produced, one in $S_{i}$ and the other in $S_{i+1}$. In other words, each cell in $S_{i}$ produces a Poisson process of cells at rate $\lambda_{i}$ into $S_{i+1}$. The death rate of a cell is $\delta_{i}$. The overall growth rate $\gamma_{i}=\mu_{i}-\delta_{i}$. All possible cell divisions and deaths are assumed independent.

The $S_{0}$ case is rather different. We assume that the subpopulations of mutants are sufficiently small compared to that of $S_{0}$, so that $S_{0}$ maintains its size, and simply produces a stream of mutants into $S_{1}$. This rate will be taken to be $\lambda_{0}$ which, unlike the other $\lambda \mathrm{s}$, will be for the whole of $S_{0}$ rather than a rate for an individual cell. We shall show the following result.

Theorem 1. If $Z_{r}(t)$ is the number of cells in $S_{r}$ at time $t$ and $\gamma_{i} \neq \gamma_{j}, i \neq j$, then

$$
\mathrm{E}\left\{Z_{r}(t)\right\}=\lambda_{0} \cdots \lambda_{r-1} \sum_{i=1}^{r} \frac{1}{\prod_{j \neq i}\left(\gamma_{i}-\gamma_{j}\right)} \frac{\mathrm{e}^{\gamma_{i} t}-1}{\gamma_{i}},
$$

and, setting $\gamma_{0}=2 \gamma_{r}$,

$$
\operatorname{var}\left\{Z_{r}(t)\right\}=\lambda_{0} \cdots \lambda_{r-1}\left(\mu_{r}+\delta_{r}\right) \sum_{i=0}^{r} \frac{1}{\prod_{j \neq i}\left(\gamma_{i}-\gamma_{j}\right)} \frac{\mathrm{e}^{\gamma_{i} t}-1}{\gamma_{i}} .
$$

We set $\gamma_{0}=2 \gamma_{r}$ purely to make the formula simple. It should be noted that the mutation rates $\left\{\lambda_{i}\right\}$ appear only in the form of their product. In Figure 2 we show the growth in the expected population sizes for a four-stage model with mutation rates into stages $1-4$ of $0.05-0.20$ and growth rates of 1.2-1.8. It is seen that, once established in a stage, the growth rates dominate.

\section{The mean and variance of the population in $S_{r}$}

We use a method employed in Kingman (1975) for the treatment of the first birth problem in branching processes. Let $B_{r 1}, \ldots, B_{r m}, \ldots$ be the birth times of cells in $S_{r}$. These include 
mutations from $S_{r-1}$ and births within $S_{r}$. Let $Z_{r}(t)$ be the number of cells in $S_{r}$ at time $t$, and define

$$
b_{r}(\theta)=\mathrm{E}\left\{\sum_{m} \mathrm{e}^{-\theta B_{r m}}\right\}=\int_{0}^{\infty} \mathrm{e}^{-\theta t} \mathrm{~d}\left\{\mathrm{E} Z_{r}(t)\right\},
$$

by Fubini's theorem.

Lemma 1. If $B_{1}, \ldots, B_{m}, \ldots$ are the birth times in $S_{r}$ of a single cell in $S_{r-1}$ born at time $t_{0}$ then

$$
\mathrm{E}\left\{\sum_{m} \mathrm{e}^{-\theta B_{m}}\right\}=\mathrm{e}^{-\theta t_{0}} \frac{\lambda_{r-1}}{\theta-\gamma_{r}} .
$$

Proof. In a birth-and-death process with birth rate $\mu$ and death-rate $\delta$ starting from a single cell at time 0 , the expected size of the population at time $t$ is $\mathrm{e}^{\gamma t}$. Thus, if the number of descendants of the cell in $S_{r-1}$ at time $s$ is $Z(s)$ then

$$
\mathrm{E}\{Z(t)\}=\int_{t_{0}}^{t} \mathrm{e}^{\gamma_{r}(t-s)} \lambda_{r-1} \mathrm{~d} s=\mathrm{e}^{\gamma_{r} t} \frac{\lambda_{r-1}}{\gamma_{r}}\left[\mathrm{e}^{-\gamma_{r} t_{0}}-\mathrm{e}^{-\gamma_{r} t}\right], \quad t>t_{0},
$$

and

$$
\mathrm{E}\left\{\sum_{m} \mathrm{e}^{-\theta B_{m}}\right\}=\int_{t_{0}}^{\infty} \mathrm{e}^{-\theta t} \lambda_{r-1} \mathrm{e}^{-\gamma_{r} t_{0}} \mathrm{e}^{\gamma_{r} t} \mathrm{~d} t=\mathrm{e}^{-\theta t_{0}} \frac{\lambda_{r-1}}{\theta-\gamma_{r}} .
$$

Lemma 2. It holds that

$$
b_{r}(\theta)=\frac{\lambda_{r-1}}{\theta-\gamma_{r}} b_{r-1}(\theta) .
$$

Proof. Let $F_{r-1, k}(t)$ be the distribution function of the birth time of the $k$ th cell in $S_{r-1}$. If this cell gives rise to cells in $S_{r}$ at times $B_{r k 1}, \ldots$ then, by Lemma 1,

$$
\mathrm{E}\left\{\sum_{m} \mathrm{e}^{-\theta B_{r k m}}\right\}=\frac{\lambda_{r-1}}{\theta-\gamma_{r}} \int_{0}^{\infty} \mathrm{e}^{-\theta t} \mathrm{~d} F_{r-1, k}(t) .
$$

As $b_{r}(\theta)=\sum_{k} \mathrm{E}\left\{\sum_{m} \mathrm{e}^{-\theta B_{r k m}}\right\}$ and $\mathrm{E}\left\{Z_{r-1}(t)\right\}=\sum_{k} F_{r-1, k}(t)$, the result follows.

Since we are assuming that the number of cells in $S_{0}$ stays constant, $\lambda_{0}$ represents the total mutation rate from $S_{0}$ and we take $b_{0}(\theta)=1$. Lemma 2 and a standard partial fraction expansion then imply the following result.

Lemma 3. If $\gamma_{i} \neq \gamma_{j}, i \neq j$, then

$$
b_{r}(\theta)=\prod_{i=1}^{r} \frac{\lambda_{i-1}}{\theta-\gamma_{i}}=\lambda_{0} \cdots \lambda_{r-1} \sum_{i=1}^{r} \frac{1}{\prod_{j \neq i}\left(\gamma_{i}-\gamma_{j}\right)} \frac{1}{\theta-\gamma_{i}} .
$$

Using (1) and Lemma 3, we obtain the first part of Theorem 1.

If $\gamma_{i} \neq \gamma_{j}, i \neq j$, then

$$
\mathrm{E}\left\{Z_{r}(t)\right\}=\lambda_{0} \cdots \lambda_{r-1} \sum_{i=1}^{r} \frac{1}{\prod_{j \neq i}\left(\gamma_{i}-\gamma_{j}\right)} \frac{\mathrm{e}^{\gamma_{i} t}-1}{\gamma_{i}} .
$$

In a birth-and-death process with birth rate $\mu$ and death rate $\delta$ starting from a single cell at time 0 , the variance of the size of the population at time $t$ is $V(t)=\gamma^{*} \mathrm{e}^{\gamma t}\left(\mathrm{e}^{\gamma t}-1\right)$, where $\gamma^{*}=(\mu+\delta) /(\mu-\delta)$ and $\gamma=\mu-\delta$. Thus, if $V_{r}(s)$ is the variance of the number of 
descendants in $S_{r}$ at time $s$ of a cell in $S_{r-1}$ born at time $t_{0}$, then

$$
\begin{aligned}
V_{r}(t) & =\gamma_{r}^{*} \int_{t_{0}}^{t}\left(\mathrm{e}^{2 \gamma_{r}(t-s)}-\mathrm{e}^{\gamma_{r}(t-s)} \lambda_{r-1}\right) \mathrm{d} s \\
& =\gamma_{r}^{*} \frac{\lambda_{r-1}}{\gamma_{r}}\left(\frac{\mathrm{e}^{2 \gamma_{r}\left(t-t_{0}\right)}-1}{2}-\left[\mathrm{e}^{\gamma_{r}\left(t-t_{0}\right)}-1\right]\right), \quad t>t_{0} .
\end{aligned}
$$

Thus,

$$
\begin{aligned}
\int_{t_{0}}^{\infty} \mathrm{e}^{-\theta t} \mathrm{~d} V_{r}(t) & =\lambda_{r-1} \gamma_{r}^{*} \int_{t_{0}}^{\infty}\left(\mathrm{e}^{-2 \gamma_{r} t_{0}} \mathrm{e}^{-\left(\theta-2 \gamma_{r}\right) t}-\mathrm{e}^{-\gamma_{r} t_{0}} \mathrm{e}^{-\left(\theta-\gamma_{r}\right) t}\right) \mathrm{d} t \\
& =\lambda_{r-1} \gamma_{r}^{*} \gamma_{r} \frac{1}{\left(\theta-2 \gamma_{r}\right)\left(\theta-\gamma_{r}\right)} \mathrm{e}^{-\theta t_{0}} .
\end{aligned}
$$

If the $k$ th particle born in $S_{r-1}$ produces a population in $S_{r}$ with variance $V_{r k}(t)$ then, setting $V_{r}(t)=\operatorname{var}\left\{Z_{r}(t)\right\}, V_{r}(t)=\sum_{k} V_{r k}(t)$ so that

$$
\int_{0}^{\infty} \mathrm{e}^{-\theta t} \mathrm{~d} V_{r}(t)=\frac{\lambda_{r-1} \gamma_{r} \gamma_{r}^{*}}{\left(\theta-2 \gamma_{r}\right)\left(\theta-\gamma_{r}\right)} \sum_{k} \int_{0}^{\infty} \mathrm{e}^{-\theta t} \mathrm{~d} F_{r-1, k}(t)=\frac{\gamma_{r} \gamma_{r}^{*}}{\theta-2 \gamma_{r}} b_{r}(\theta),
$$

as in the proof of Lemma 2. By setting $\gamma_{0}=2 \gamma_{r}$ we can express the formula for the variance in much the same form as for the mean. Using the above equation and Lemma 3, we obtain the second part of Theorem 1.

If $\gamma_{i} \neq \gamma_{j}, i \neq j$, and $\gamma_{0}=2 \gamma_{r}$, then

$$
\operatorname{var}\left\{Z_{r}(t)\right\}=\lambda_{0} \cdots \lambda_{r-1}\left(\mu_{r}+\delta_{r}\right) \sum_{i=0}^{r} \frac{1}{\prod_{j \neq i}\left(\gamma_{i}-\gamma_{j}\right)} \frac{\mathrm{e}^{\gamma_{i} t}-1}{\gamma_{i}},
$$

since $\gamma_{r} \gamma_{r}^{*}=\mu_{r}+\delta_{r}$.

\section{The probability $S_{r}$ is occupied}

In this section we will calculate the probability that the mutation chain has reached $S_{r}$ by time $t$. In order to solve the differential equations, we have assumed that the death rate $\delta=0$. We will start by calculating the probability that a cell born at time 0 in $S_{r-1}$ has offspring in $S_{r}$, and then we will work backwards until we can calculate this probability for cells born in $S_{0}$. We will distinguish two ways in which descendants of the cell in $S_{i}$ arrive in $S_{r}$. First, they can arrive by direct mutation from the cell in $S_{i}$ to $S_{i+1}$. This stream of cells is a Poisson process at rate $\lambda_{i}$. Second, they can arrive as mutations from $S_{i}$ to $S_{i+1}$ from descendants of the original cell within $S_{i}$, that is, from the birth process in $S_{i}$ of that cell. What happens from $S_{i+1}$ is not relevant to this definition, only the manner in which descendants arrive in $S_{i+1}$. For $i<r$, define

- $C_{i}(t)$ to be the probability that a cell born in $S_{i}$ at time 0 has no descendants in $S_{r}$ at time $t$ arising from direct mutations from the cell to $S_{i+1}$,

- $B_{i}(t)$ to be the probability that a cell born in $S_{i}$ at time 0 has no descendants in $S_{r}$ at time $t$ arising from mutations to $S_{i+1}$ from descendants of the cell in $S_{i}$.

We should note that the probability that a cell born in $S_{i}$ at time 0 has no descendants in $S_{r}$ at time $t$ equals $C_{i}(t) B_{i}(t)$. 
We condition on the first birth to the cell in $S_{i}$ at time $s$. There are then two cells. The probability that the new cell has no offspring equals $B_{i}(t-s) C_{i}(t-s)$. Any descendants of this cell are descendants of the birth process from the original cell. However, only descendants from future births to the original cell count as descendants of the birth process from the original cell. We thus have

$$
\begin{aligned}
B_{i}(t) & =\int_{0}^{t} B_{i}(t-s) C_{i}(t-s) B_{i}(t-s) \mu_{i} \mathrm{e}^{-\mu_{i} s} \mathrm{~d} s+\mathrm{e}^{-\mu_{i} t} \\
& =\mu_{i} \mathrm{e}^{-\mu_{i} t} \int_{0}^{t} B_{i}^{2}(u) C_{i}(u) \mathrm{e}^{\mu_{i} u} \mathrm{~d} u+\mathrm{e}^{-\mu_{i} t} .
\end{aligned}
$$

Dropping the subscript $i$ for the moment and multiplying both sides by $\mathrm{e}^{\mu t}$ and differentiating, we obtain

$$
[\mu B+\dot{B}] \mathrm{e}^{\mu t}=\mu C B^{2} \mathrm{e}^{\mu t} .
$$

Setting $D=1 / B$ and multiplying each side by $\mathrm{e}^{-\mu t}$ gives

$$
[\mu D-\dot{D}] \mathrm{e}^{-\mu t}=\mu \mathrm{e}^{-\mu t} C=\frac{\mathrm{d}}{\mathrm{d} t}\left[\mathrm{e}^{-\mu t} D\right] \quad \Longrightarrow \quad-\mathrm{e}^{-\mu t} D=\int_{0}^{t} \mu \mathrm{e}^{-\mu s} C \mathrm{~d} s-1,
$$

since $D(0)=1 / B(0)=1$. Conditioning on the first mutation from the cell to $S_{i+1}$ we obtain

$$
\begin{aligned}
C_{i}(t) & =\int_{0}^{t} B_{i+1}(t-s) C_{i+1}(t-s) C_{i}(t-s) \lambda_{i} \mathrm{e}^{-\lambda_{i} s} \mathrm{~d} s+\mathrm{e}^{-\lambda_{i} t} \\
& =\lambda_{i} \mathrm{e}^{-\lambda_{i} t} \int_{0}^{t} B_{i+1}(u) C_{i+1}(u) C_{i}(u) \mathrm{e}^{\lambda_{i} u} \mathrm{~d} u+\mathrm{e}^{-\lambda_{i} t} .
\end{aligned}
$$

Multiplying both sides by $\mathrm{e}^{\lambda_{i} t}$ and differentiating we obtain

$$
\mathrm{e}^{\lambda_{i} t}\left[\lambda_{i} C_{i}+\dot{C}_{i}\right]=\lambda_{i} \mathrm{e}^{\lambda_{i} t} B_{i+1} C_{i+1} C_{i} \Longrightarrow \frac{\mathrm{d}}{\mathrm{d} t} \ln C_{i}=\left[\lambda_{i} B_{i+1} C_{i+1}-\lambda_{i}\right] .
$$

Solving equations (2) and (3) gives the following result.

\section{Theorem 2. It holds that}

$$
\begin{gathered}
B_{i}(t)=\frac{\mathrm{e}^{-\mu_{i} t}}{1-\int_{0}^{t} \mu_{i} \mathrm{e}^{-\mu_{i} s} C_{i}(s) \mathrm{d} s}, \\
C_{i}(t)=\mathrm{e}^{-\lambda_{i} t} \exp \left(\lambda_{i} \int_{0}^{t} B_{i+1}(s) C_{i+1}(s) \mathrm{d} s\right) .
\end{gathered}
$$

\section{References}

Armitage, P. And Doll, R. (1957). A two-stage theory of carcinogenesis in relation to the age distribution of human cancer. British J. Cancer 11, 161-169.

Connolly, R. B. And Kimbell, J. S. (1994). Simulation of cell growth governed by stochastic processes: application to clonal growth cancer models. Toxicology Appl. Pharmacology 124, 284-295.

Kingman, J. F. C. (1975). The first birth problem for an age-dependent branching process. Ann. Prob. 3, $790-801$.

Portier, C. J., Kopp-Schneider, A. And Sherman, C. D. (1996). Calculating tumor incidence rates in stochastic models. Math. Biosci. 135, 129-146.

Portier, C. J., Sherman, C. D. And Kopp-Schneider, A. (2000). Multistage stochastic models of the cancer process: a general theory for calculating tumor incidence. Stoch. Environ. Res. Risk Assess. 14, 173-179.

Zheng, Q. (2008). Stochastic multistage cancer models: a fresh look at an old approach. In Handbook of Cancer Models with Applications, World Scientific, Singapore, pp. 25-44. 